\title{
RB1 Positive
}

National Cancer Institute

\section{Source}

National Cancer Institute. RB1 Positive. NCI Thesaurus. Code C128842.

Indicates that RB1 expression has been detected in a sample. 